\title{
Ultrathin Gate Oxides Prepared by Tensile-Stress Oxidation in Tilted Cathode Anodization System
}

\author{
Chih-Ching Wang, Tsung-Hung Li, Kai-Chieh Chuang, and Jenn-Gwo Hwu ${ }^{\mathrm{z}}$ \\ Department of Electrical Engineering, Graduate Institute of Electronics Engineering, National Taiwan
} University, Taipei, Taiwan

\begin{abstract}
The ultrathin gate oxides prepared by mechanical tensile-stress oxidation in the tilted cathode anodization system were studied. By bending the silicon wafer during the anodization process and releasing stress in the final stage, we applied mechanical tensile stress onto the silicon wafer to overcome the lattice mismatch between $\mathrm{Si}$ and $\mathrm{SiO}_{2}$. As a result of external mechanical stress in the gate oxide, different electrical characteristics of the metal-oxide-superconductor capacitors are discovered. Flatband voltages of the experimental samples with tensile-stress shift positively due to bandgap narrowing effect. It was observed that the quality and reliability of $\mathrm{SiO}_{2}$ grown by tensile-stress oxidation are significantly improved.
\end{abstract}

(c) 2007 The Electrochemical Society. [DOI: 10.1149/1.2823494] All rights reserved.

Manuscript submitted July 12, 2007; revised manuscript received November 6, 2007. Available electronically December $27,2007$.

As the feature size of complementary metal-oxidesuperconductor (CMOS) devices is scaling down, the ultrathin gate dielectric with an equivalent oxide thickness (EOT) of $\sim 2 \mathrm{~nm}$ is indispensable for nanoscale technology. At the same time, the interfacial transition region's fraction becomes larger of the total oxide layer. Conventional gate dielectrics (i.e., $\mathrm{SiO}_{2}$ ) encounter the severe issue that the gate leakage current is approaching a critical level. High gate leakage current will cause CMOS devices to lose ideal electrical characteristics or cause reliability problems. Although the $\mathrm{Si} / \mathrm{SiO}_{2}$ interface is excellent for most cases, the lattice constant of $\mathrm{Si}$ does not match that of $\mathrm{SiO}_{2}$, whereas $\mathrm{SiO}_{2}$ has a greater lattice constant. The lattice constant mismatch will form some bonding defects at the $\mathrm{Si}-\mathrm{SiO}_{2}$ interface, such as $\mathrm{Si}-\mathrm{Si}$ stretched bonds and Si dangling bonds. These defects would turn into interface traps and suboxide transition regions, ${ }^{1}$ which degrade the electrical characteristics, and would cause higher gate leakage current. ${ }^{2}$

We managed to bend the silicon wafer to overcome lattice constant mismatch between $\mathrm{Si}$ and $\mathrm{SiO}_{2}$ during the oxidation process. In microview, one side of the Si lattice on the surface will then sustain tensile stress so that the Si lattice will stretch out and become wider than usual. When the oxidant reacts with $\mathrm{Si}$, the interface will be more perfect because of the less lattice constant mismatch. Applying external mechanical stress on a silicon wafer has been explored in the conventional thermal oxidation process. ${ }^{3}$ For a silicon wafer sustaining tensile stress, the linear rate constant of the Deal-Grove model was found to be deformation dependent and the parabolic constant was stress dependent. ${ }^{3}$ Furthermore, oxides as gate dielectrics of excellent quality can be fabricated by anodization, ${ }^{4,5}$ which is a room-temperature oxidation process in deionized water (electrolyte). We attempt to apply mechanical tensile stress through the anodization process; thus, thermal stress effect can be avoided. In this work, electrical characteristics of the anodized oxides grown with applied tensile stress are compared to those of the control sample.

\section{Experimental}

The 3 in. boron-doped p-type silicon (100) wafers with a resistivity of $1-10 \Omega \mathrm{cm}$ and a doping concentration of $5 \times 10^{15} \mathrm{~cm}^{-3}$ were used as the substrate of the MOS (p) capacitors. After standard RCA cleaning, oxides were grown through the anodization process. Postoxidation annealing was carried out in 100 Torr $\mathrm{N}_{2}$ at $800^{\circ} \mathrm{C}$ for $15 \mathrm{~s}$ by rapid thermal process equipment. Following the thermal evaporation of $\mathrm{Al}$ film as the gate electrode, the MOS capacitors of size $150 \times 150 \mu \mathrm{m}$ were formed by conventional photolithography and wet etching. Finally, Al with $1 \%$ silicon was thermally evaporated on the back side of wafer to serve as the back contact.

Figure 1 shows the setup of the anodization system with tensile-

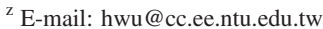

stress application. A bar butted the back side of the wafer so that the other side, which was in front of the Pt electrode (cathode), would then sustain tensile stress. The schematic plot of the measured area along with the diameter of the silicon wafer with applied mechanical tensile stress is also shown in Fig. 2. The anodization process was carried out for $10 \mathrm{~min}$. During the last $2 \mathrm{~min}$, we gradually released the mechanical tensile stress applied onto the wafer. In order to fix the position of the nonstress sample without applied stress, the bar still "slightly touched" the wafer. The Pt electrode was tilted so that oxides with various thicknesses can be grown on a single wafer as shown in Fig. 3. In this way, oxide thicknesses distributed from 1.8 to $3.0 \mathrm{~nm}$ could be acquired on one wafer. The thickness distribution measured by ellipsometry is shown in Fig. 4. If a specified horizontal position is chosen, then the oxide thickness will gradually change (thinner or thicker) in the vertical direction. The tensilestress sample also shows a similar distribution.

In this experiment, the capacitance-voltage $(C-V)$ and current density-voltage $(J-V)$ curves were measured by an HP4280 precision inductance, capacitance, resistance (LCR) meter and an HP4140B pA meter, respectively. The EOT was extracted by fitting the two-frequency, three-element model corrected $C-V$ curve under the consideration of quantum mechanical effect. ${ }^{6,7}$ Frequencies of $100 \mathrm{k}$ and $1 \mathrm{M} \mathrm{Hz}$ are taken for $C-V$ correction. During the reliability test, the stressing current was injected by a Keithley 220 programmable current source.

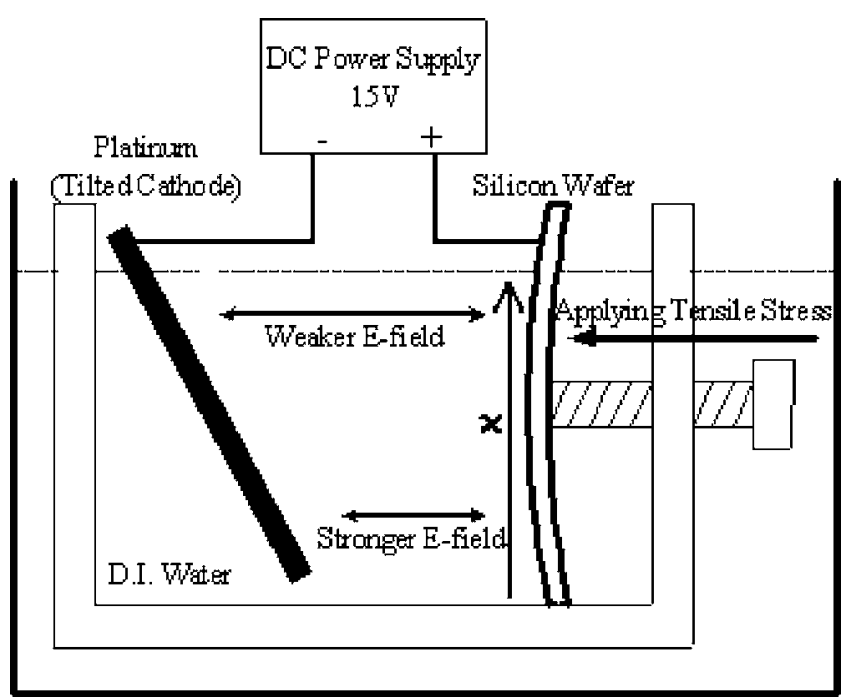

Figure 1. Experimental setup of the tilted cathode anodization system. External mechanical stress was exerted on the silicon wafer. 


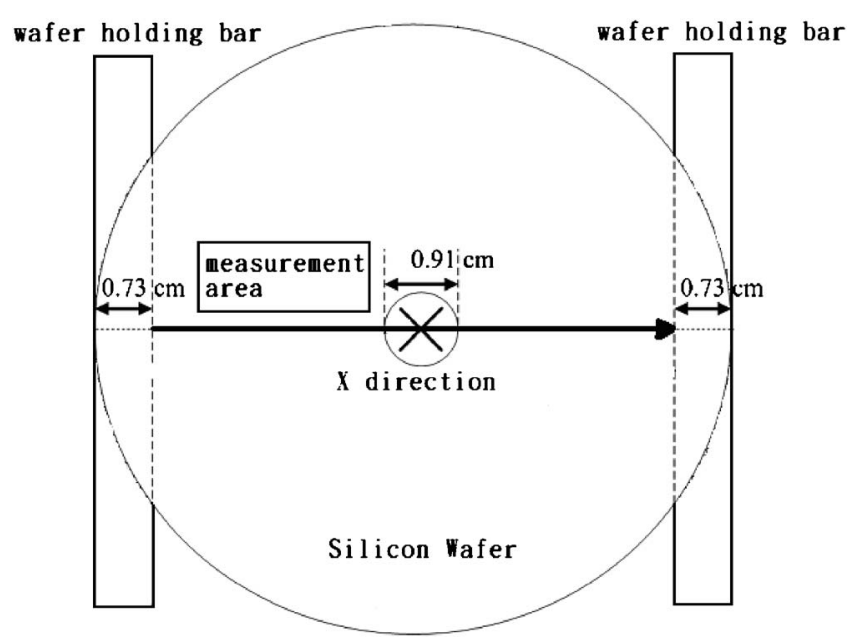

Figure 2. Schematic view of the measured area along the diameter.

\section{Results and Discussion}

Electrical characteristics of tensile-stress sample.- $C$ - $V$ curves of the nonstress and tensile stress MOS (p) capacitors with distrib-

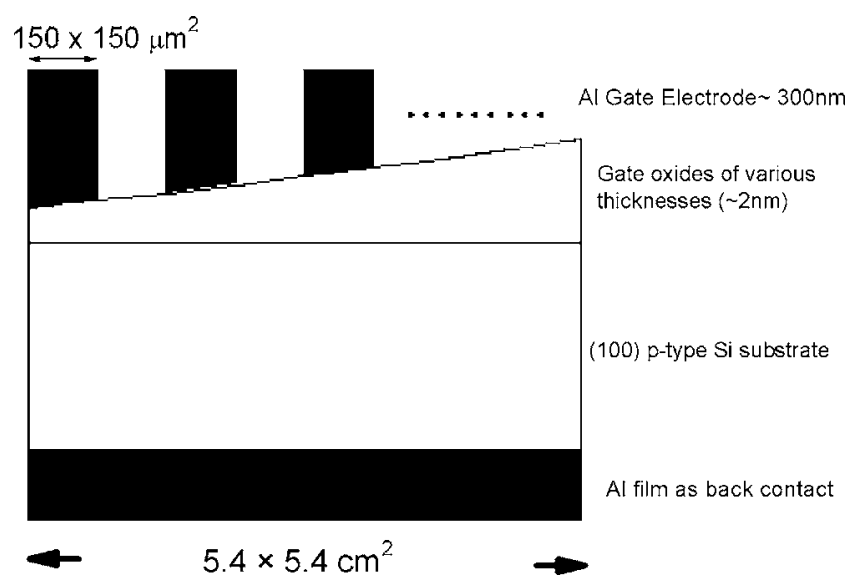

Figure 3. Schematic diagrams of device structure prepared by the tilted cathode anodization system.

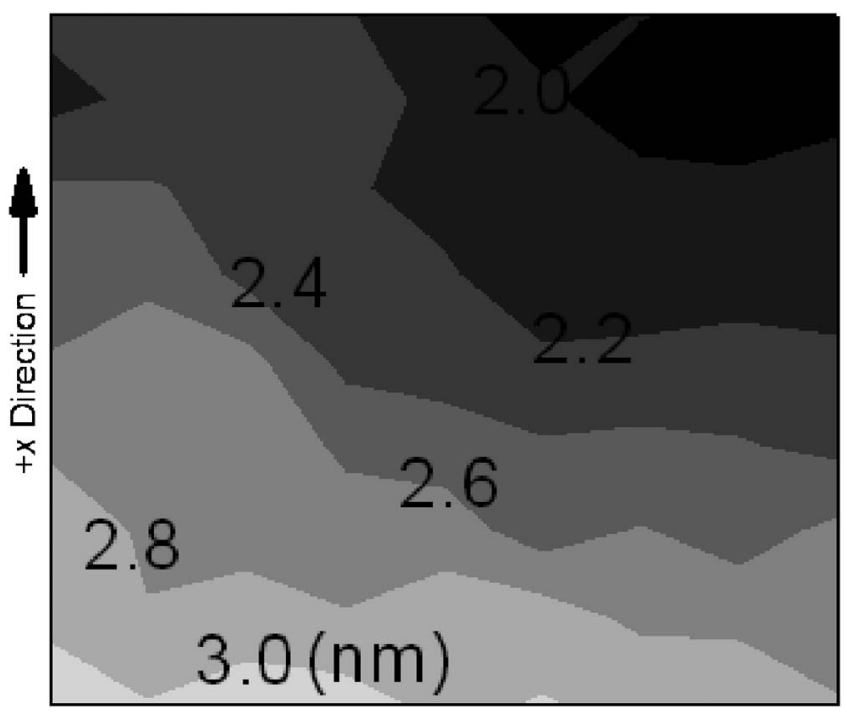

Figure 4. Oxide thickness distribution measured by ellipsometery.

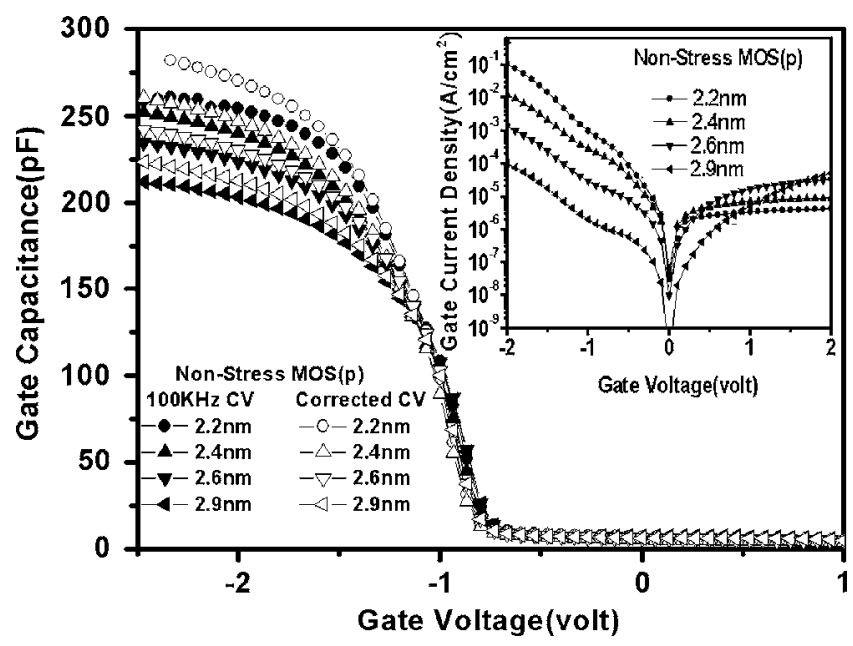

Figure 5. The $100 \mathrm{kHz}$ and two frequency corrected $C$ - $V$ curves of the nonstress MOS (p) capacitors with various EOTs. The inset is the corresponding $J-V$ curves.

uted thickness are shown in Fig. 5 and 6, respectively. The insets show the corresponding $J-V$ curves of both samples. Under the comparisons of the nonstress and tensile-stress samples, we can observe that tensile-stress devices exhibit much more perfect $C$ - $V$ curve characteristics than nonstress devices. Under EOT $=2.2 \mathrm{~nm}$, we can see that the tensile-stress sample still exhibits good $C$ - $V$ curves without capacitance attenuation, while the nonstress one does not. Figure 7 shows the gate leakage current density vs EOT relationship at $V_{\mathrm{G}}=V_{\mathrm{FB}}-1 V$. The gate leakage current of the tensile-stress sample is much smaller than that of the nonstress sample at $V_{\mathrm{G}}$ $=V_{\mathrm{FB}}-1 V$. It is noted that under $V_{\mathrm{G}}=V_{\mathrm{FB}}-1 \mathrm{~V}$, i.e., accumulation region, the leakage current is strongly related to the gate dielectrics quality. With the benefit of tilted anodization, the EOT ranging between 2.2 and $2.9 \mathrm{~nm}$ can be obtained. It is obvious that the tensile-stress sample has lower gate leakage current than the nonstress sample by several times at the same EOT. This is a piece of evidence suggesting that the oxide quality is significantly improved by this technique, mentioned above. Furthermore, the effect is supposed to be more evident for thinner films because the presence of defects in the suboxide transition regions becomes much more crucial to the oxide quality.

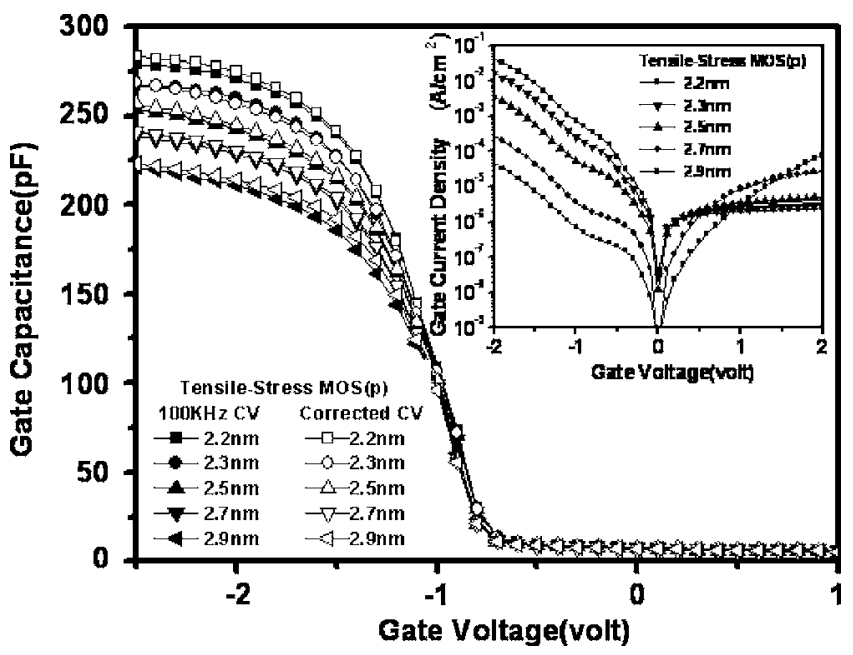

Figure 6. The $100 \mathrm{kHz}$ and two frequency corrected $C$ - $V$ curves of the tensile-stress MOS (p) capacitors with various EOTs. The inset is the corresponding $J-V$ curves. 


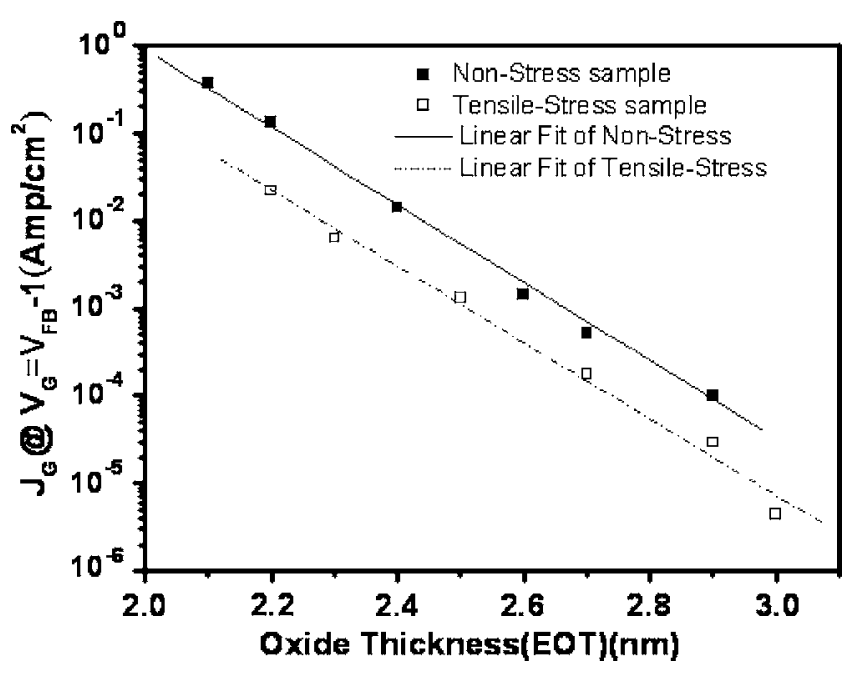

Figure 7. Relationship between gate leakage current density vs EOT for nonstress and tensile-stress $\operatorname{MOS}(\mathrm{p})$ at $V_{\mathrm{G}}=V_{\mathrm{FB}}-1 V$.

Figure 8 shows the typical $C$ - $V$ curves of the nonstress and tensile-stress MOS (p) capacitors. It is found that the flatband voltage $\left(V_{\mathrm{FB}}\right)$ of the tensile-stress sample shifted positively to $-0.82 \mathrm{~V}$ around while that of nonstress $C$ - $V$ curve is very close to the ideal value $-0.89 \mathrm{~V}$. Figure 9 shows the $V_{\mathrm{FB}}$ vs accumulation gate capacitance for nonstress and tensile-stress samples. The $V_{\mathrm{FB}}$ of the tensile-stress sample (i.e., $V_{\mathrm{FB}}=-0.80$ to $-0.82 \mathrm{~V}$ ) are more positive than those of the nonstress ones, i.e., -0.85 to $-0.88 \mathrm{~V}$. There might be several reasons leading to flatband voltage shifts of tensilestress MOS capacitors, such as interface states, fixed oxide charges, and stress effects. In order to examine if the stress did harm to the $\mathrm{Si}-\mathrm{SiO}_{2}$ interface, the interface trap-induced capacitance $\left(C_{\mathrm{it}}\right)$ is evaluated, respectively, according to the capacitance difference at $V_{\mathrm{FB}}$ between 100 and $1 \mathrm{kHz}$ by the modified hi-lo frequency method describled in Ref. 8. The result reports that under different oxide thickness, $C_{\mathrm{it}}$ is calculated to be $31.0-34.8 \mathrm{pF}$ for the tensile-stress samples, while $27.7-30.6 \mathrm{pF}$ for the nonstress ones. The discrepancy is negligibly small, which implies that bending the wafer does no harm to the $\mathrm{Si}-\mathrm{SiO}_{2}$ interfacial property. From another point of view

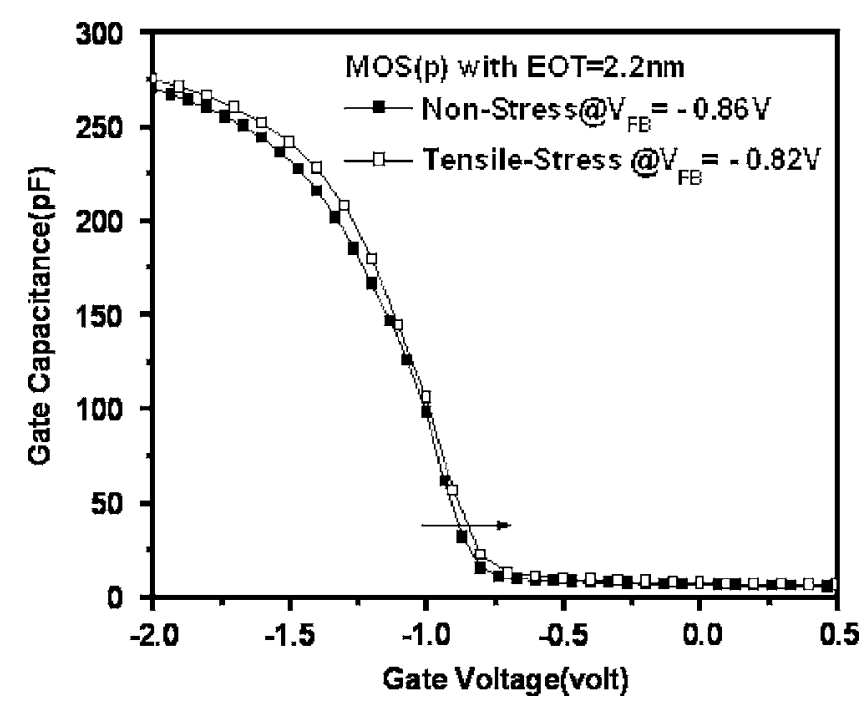

Figure 8. Typical $C$ - $V$ curves of the nonstress and tensile-stress samples.

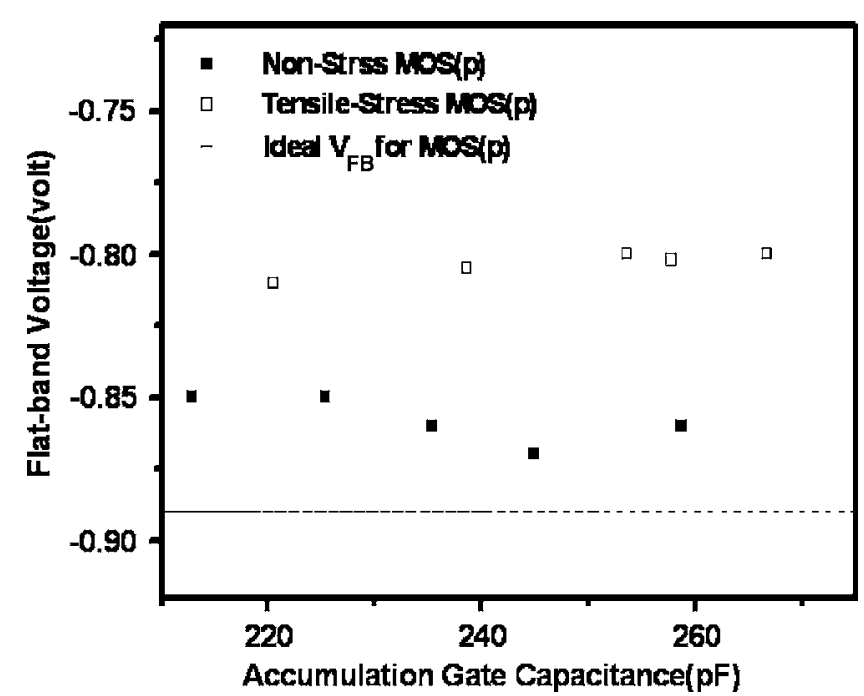

Figure 9. Flatband voltages of the nonstress and tensile-stress samples with distributed thickness.

$$
V_{\mathrm{FB}}=W_{\mathrm{ms}}-\frac{Q_{\text {eff }}}{C_{\mathrm{ox}}}
$$

where the $V_{\mathrm{FB}}$ is the flatband voltage, $\mathrm{W}_{\mathrm{ms}}$ is the work function difference, $C_{\mathrm{ox}}$ is the gate capacitance, and $Q_{\text {eff }}$ is the effective oxide charge, including interfacial charge and the fixed oxide charge. From Fig. 9, we can calculate the effective oxide charge density. The values of effective oxide charge density are $6.2-7.2 \times 10^{11} \mathrm{~cm}^{-2}$ for tensile-stress samples and $1.8-3.51 \times 10^{11} \mathrm{~cm}^{-2}$ for nonstress samples, respectively. The difference is more than two times. However, because the interfacial quality of tensile-stress sample is close to that of the nonstress sample, the tensile-stress sample is supposed to have a similar fixed oxide charge density. Thus, we would attribute the discrepancy to the band-narrowing effect. The charge of the flatband voltage in MOS due to mechanical stress $\varepsilon$ is generally given by

$$
\Delta V_{\mathrm{FB}}(\varepsilon)=\Delta \phi_{\mathrm{m}}(\varepsilon)-\Delta \phi(\varepsilon)-\frac{Q_{\mathrm{ox}}}{C_{\mathrm{ox}}}
$$

where $\phi_{\mathrm{m}}$ and $\phi_{\mathrm{s}}$ are the work functions of metal and semiconductor, respectively. Assuming that the stress-induced oxide charge variation could be neglected, Eq. 2 can be simplified as

$$
\Delta V_{\mathrm{FB}}=\Delta \phi_{\mathrm{m}}(\varepsilon)-\Delta \phi_{\mathrm{s}}(\varepsilon)
$$

It is noted that the change of metal work function $\phi_{\mathrm{m}}(\varepsilon)$ is related to the charge of the volume of metal. ${ }^{9}$ In this work, $\Delta \phi_{\mathrm{m}}$ is neglected because the stress in $\mathrm{Al}$ metal gate is small due to the $\mathrm{Al}$ formation after tensile-stress oxidation preparation. Therefore, the change of the flatband voltage due to tensile-stress oxidation is expected to be mainly due to the change of the Fermi level and the valence band of the semiconductor. Figure 10 shows the illustration of the energy band diagram with silicon bandgap narrowing effect. The $E_{\mathrm{F}}$ of silicon of the tensile-stress MOS (p) shifted upward, so that the $\phi_{\mathrm{s}}$ is reduced and, in turn, introduces a less negative $\phi_{\mathrm{m}}$. Therefore, a decrease of $\phi_{\mathrm{m}_{\mathrm{s}}}$ in negative value is observed. This could be the main possible reason which leads to flatband shifts.

Reliability of tensile-stress sample.- Furthermore, the cumulative distribution of absolute gate voltage deviation $\left(\left|\triangle V_{\mathrm{G}}\right|\right)$ of nonstress and tensile-stress samples after constant current stress is shown in Fig. 11. The MOS capacitors were stressed under a constant current density of $-10 \mathrm{~mA} / \mathrm{cm}^{2}$ for $15 \mathrm{~s}$. The inset shows the comparisons of the monitored gate voltage vs stress time for nonstress and tensile-stress samples under EOT of $2.6 \mathrm{~nm}$. We observe 


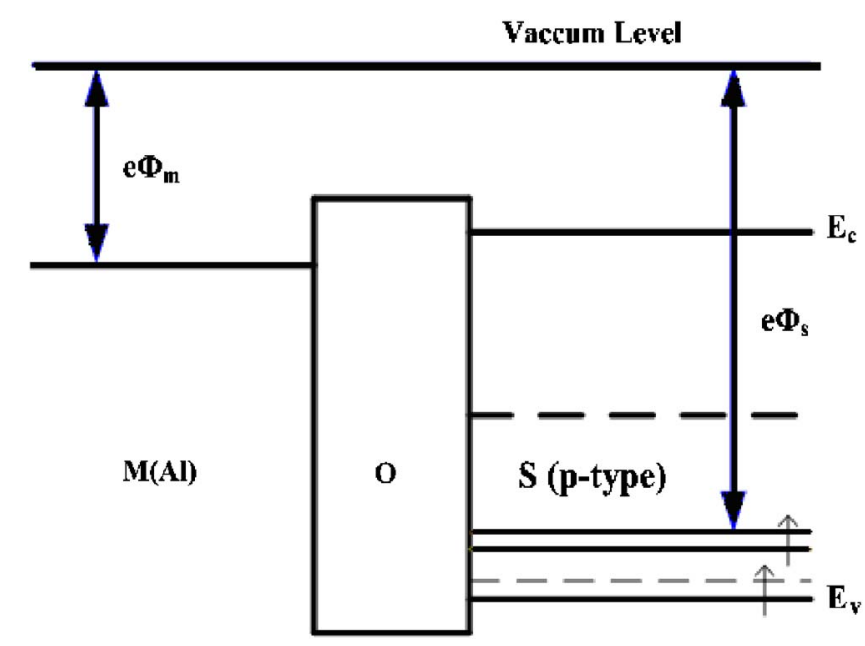

Figure 10. Band diagram of MOS (p) capacitors with silicon bandgap narrowing effect due to tensile stress.

that the monitored gate voltages of the tensile-stress sample tend to change less under the same constant current stressing, which indicates less electron trapping. Moreover, we also observe that thicker gate oxides exhibit a similar trend but a bigger voltage deviation. The tensile-stress sample shows smaller gate voltage deviation than the nonstress sample, which implies that the sample grown with applied mechanical tensile stress during anodic oxidation exhibits better reliability.

Finally, we conducted a reliability test to compare the timedependent dielectric breakdown of the nonstress and tensile-stress samples. It was reported that for thin oxides, breakdown is related to the buildup of neutral trap density. ${ }^{10}$ This "wear-out" model of oxide breakdown has been investigated for a long time. ${ }^{11-13}$ It is believed that traps build up in the oxide when the devices are stressed. When the trap density reaches a critical level, a conduction path is formed and the oxide goes to breakdown. ${ }^{12}$ The reliability tests focus on the time to breakdown $\left(T_{\mathrm{BD}}\right)$ for the nonstress and tensile-stress samples. Figure 12 shows the Weibull plot of $T_{\mathrm{BD}}$ for ten devices of

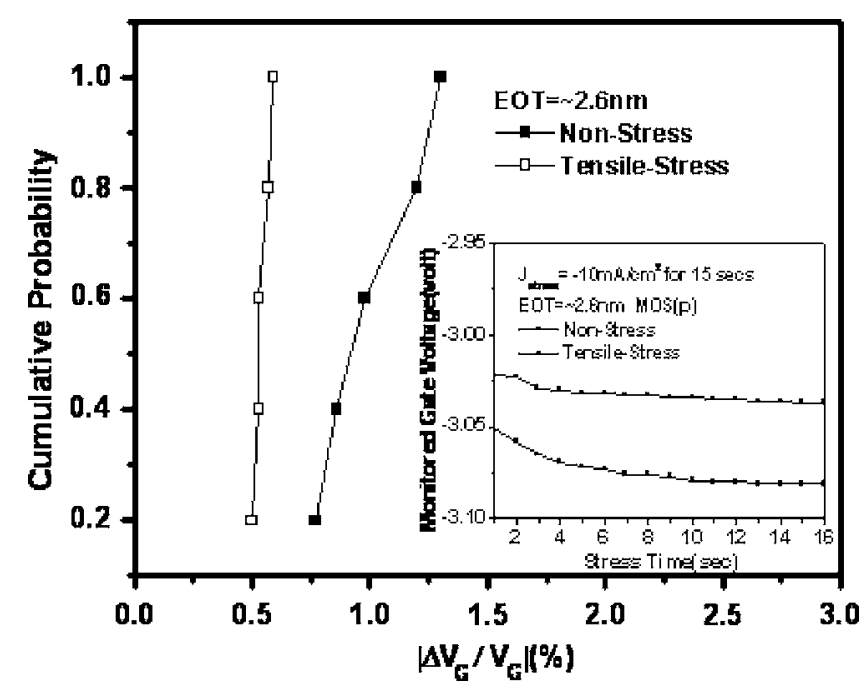

Figure 11. Cumulative plots of the absolute gate voltage deviations for nonstress and tensile-stress samples after constant current stress $-10 \mathrm{~mA} / \mathrm{cm}^{2}$ for $15 \mathrm{~s}$. The inset shows the comparisons of the monitored gate voltage vs stress time for nonstress and tensile-stress samples.

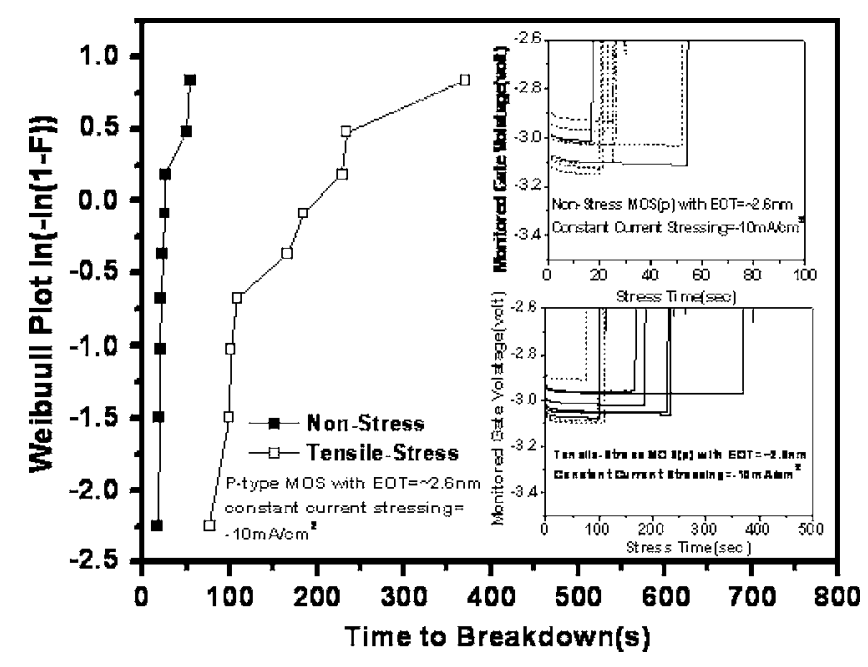

Figure 12. The Weibull plots of the $T_{\mathrm{BD}}$ for the nonstress and tensile-stress samples. The insets show the monitored gate voltage vs stress time for nonstress and tensile-stress samples under constant current stress $-10 \mathrm{~mA} / \mathrm{cm}^{2}$.

$\operatorname{MOS}(\mathrm{p})$ capacitors with EOT $=\sim 2.6 \mathrm{~nm}$ stressed under constant current of $-10 \mathrm{~mA} / \mathrm{cm}^{2}$. The insets show the monitored gate voltage vs the stress time for nonstress and tensile-stress samples. We observe that the endurance time of the tensile-stress sample is longer than that of the nonstress samples. It indicates that the timedependent dielectric breakdown characteristic of tensile-stress sample is improved.

\section{Conclusion}

In this work, ultrathin gate oxides prepared by mechanical tensile-stress oxidation during anodization process to reduce the gate leakage current are demonstrated. The flatband voltage shifts of the samples grown with applied mechanical tensile stress are observed and the possible reason might be caused by silicon bandgap narrowing effect. The reliability of the tensile-stress oxidation sample is also much improved. This is important for the preparation of highquality ultrathin gate oxides.

\section{Acknowledgments}

This work was supported by the National Science Council, ROC, under contract no. NSC96-2628-E-002-246-MY3.

National Taiwan University assisted in meeting the publication costs of this article.

\section{References}

1. E. H. Nicollian and J. R. Brews, MOS Physics and Technology, p. 212, Wiley, New York (1981).

2. P. M. Lenahan, J. J. Mele, J. P. Campbell, A. Y. Kang, R. K. Lowry, D. Woodbury, S. T. Liu, and R. Weimer, IEEE International Reliability Physics Symposium, pp. 150-155, May (2001)

3. J. Y. Yen and J. G. Hwu, J. Appl. Phys., 89, 3027 (2001).

4. G. C. Jain, A. Prasad, and B. C. Chakravarty, J. Electrochem. Soc., 126, 89 (1979).

5. M. Grecea, C. Rotaru, N. Nastase, and G. Craciun, J. Mol. Struct., 480-481, 607 (1999).

6. K. J. Yang and C. Hu, IEEE Trans. Electron Devices, 46, 1500 (1999).

7. K. Yang, Y. C. King, and C. Hu, in Digest of Technical Papers, Symposium on VLSI Technology, pp. 77-78, IEEE (1999).

8. S. W. Huang and J. G. Hwu, IEEE Trans. Electron Devices, 50, 1658 (2003).

9. N. W. Ashcroft and N. D. Mermin, Solid State Physics, p. 32, Holt, Rinehart, and Winston, New York (1976)

10. R. Degraeve, G. Groeseneken, R. Bellens, M. Depas, and H. E. Maes, Tech. Dig. Int. Electron Devices Meet., 1995, 863.

11. E. Harari, J. Appl. Phys., 49, 2478 (1978).

12. B. Rico, M. Y. Azbel, and M. H. Brodsky, Phys. Rev. Lett., 51, 1795 (1983).

13. Y. Nissan-Cohen, J. Shappir, and D. Frohman-Bentchkowsky, J. Appl. Phys., 60, 2024 (1986). 